\title{
Towards More Efficient Logic Blocks By Exploiting Biconditional Expansion
}

\author{
Pierre-Emmanuel Gaillardon, EPFL \\ Gain Kim, EPFL \\ Xifan Tang, EPFL \\ Luca Amarù, EPFL \\ Giovanni De Micheli, EPFL
}

Nowadays, Field Programmable Gate Arrays (FPGA) exploit Look-Up Tables (LUTs) to generate logic functions. A $K$-input LUT can implement any Boolean functions with $K$ inputs. Thanks to this flexibility, LUTs remained conceptually unchanged in FPGAs, only the number of inputs increased in time. Unfortunately, the flexibility does not come for free and LUTs have non-negligible costs in both circuit-level performances (large number of memories, area or delay penalties) and logic-level capabilities (limited fan-out). Here, we propose an FPGA fabric based on two novel logic blocks. First, we introduce a new LUT design showing reduced power consumption with no sacrifice in the logic flexibility. Then, we present a block suited to arithmetic functions but preserving enough versatility to implement general logic functions. The two blocks are supported by a recently introduced logic representation called Biconditional Binary Decision Diagrams (BBDDs). Using architectural-level benchmarking, we showed that an FPGA architecture exploiting the novel blocks performs significantly better than current state-of-the-art FPGA architectures at $40 \mathrm{~nm}$ technological node over a large set of test circuits. While reducing the power consumption of MCNC big20 benchmarks by $29 \%$, the proposed architecture is able to efficiently implement arithmetic circuits as compared to its traditional LUT-based FPGA counterpart. For instance, a 256bit adder can be realized with a $43 \%$ gain in area $\times$ delay product. While considering large general and arithmetic logic benchmarks, we observe, on average, $4 \%, 3 \%$ and $10 \%$ improvements in area, delay and power respectively.

ACM Categories \& Descriptors: H.3.6 Programmable logic elements

Keywords: FPGA; Arithmetic functions; BBDD; Logic Element

DOI: http://dx.doi.org/10.1145/2684746.2689100 